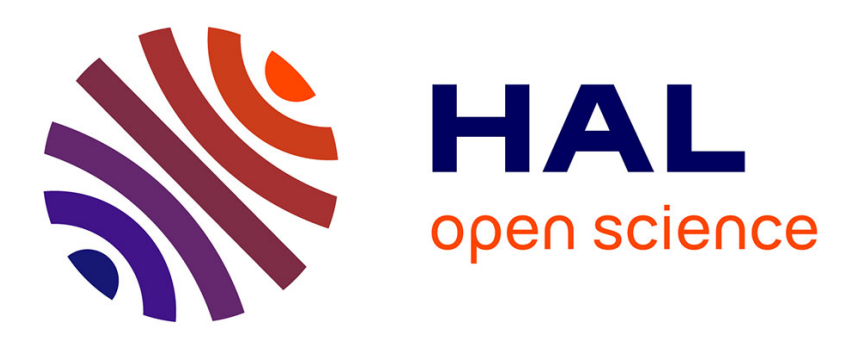

\title{
DET2007 Special Issue: A modelling approach for dedicated machinery to support the effects of mass customization
}

\author{
Jason Anthony Matthews
}

\section{- To cite this version:}

Jason Anthony Matthews. DET2007 Special Issue: A modelling approach for dedicated machinery to support the effects of mass customization. International Journal of Computer Integrated Manufacturing, 2009, 22 (11), pp.1000-1011. 10.1080/09511920802527608 . hal-00528088

\section{HAL Id: hal-00528088 \\ https://hal.science/hal-00528088}

Submitted on 21 Oct 2010

HAL is a multi-disciplinary open access archive for the deposit and dissemination of scientific research documents, whether they are published or not. The documents may come from teaching and research institutions in France or abroad, or from public or private research centers.
L'archive ouverte pluridisciplinaire HAL, est destinée au dépôt et à la diffusion de documents scientifiques de niveau recherche, publiés ou non, émanant des établissements d'enseignement et de recherche français ou étrangers, des laboratoires publics ou privés. 


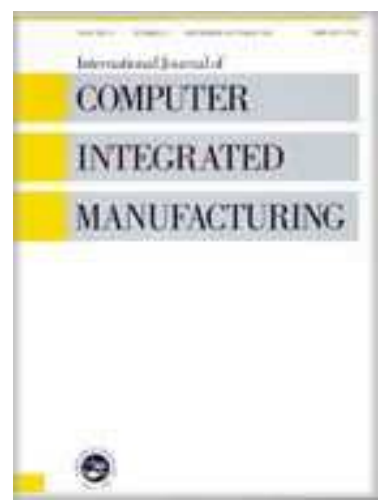

DET2007 Special Issue: A modelling approach for dedicated machinery to support the effects of mass customization

\begin{tabular}{|r|l|}
\hline Journal: & International Journal of Computer Integrated Manufacturing \\
\hline Manuscript ID: & TCIM-2008-IJCIM-0032.R1 \\
\hline Manuscript Type: & Special Issue Paper \\
\hline $\begin{array}{r}\text { Date Submitted by the } \\
\text { Author: }\end{array}$ & 18-Sep-2008 \\
\hline Complete List of Authors: & $\begin{array}{l}\text { MATTHEWS, JASON; UNIVERISTY OF BATH, MECHANICAL } \\
\text { ENGINEERING }\end{array}$ \\
\hline Keywords: & $\begin{array}{l}\text { DESIGN OF PRODUCTION SYSTEMS, MASS CUSTOMIZATION, } \\
\text { PROCESS MODELLING }\end{array}$ \\
\hline Keywords (user): & manufacturing systems evolution, product variation \\
\hline \multicolumn{2}{|l}{} \\
\hline
\end{tabular}

\section{s ScholarONE" \\ Manuscript Central}




\title{
A modelling approach for dedicated machinery to support the effects of mass customization
}

\author{
J Matthews ${ }^{1}$, B Singh ${ }^{1}$, A Delandes ${ }^{2}$, G Mullineux $^{1}$ and A. J Medland ${ }^{1}$ \\ ${ }^{1}$ Department of Mechanical Engineering, University of Bath, BA2 7AY \\ ${ }^{2}$ Universite de Technologie, 60206, Compiegne, Cedex
}

\begin{abstract}
In recent years the strategy of mass customization has become being increasingly adopted as manufacturers seek to exploit market trends for greater product variety and individualisation. The implications of changing to mass customization practice are considerable, specifically for the equipment producing the product. It is common practice within manufacturing companies to rely on trial-and-error approaches to develop their existing processing equipment for new or variant products. The approach presented in this paper employs the capability of a modelling environment to represent and simulate variations in machine design configuration and assess their ability to process variant products. The approach is demonstrated on an example from the packaging industry.
\end{abstract}

Keywords: Process modelling, manufacturing systems evolution, product variation, mass customization 
Dear editor,

First I would like to thank you and the reviewer for their efforts.

The Revisions:

1. Figures and their numbering have been modified from 'figure 6' onwards.

Thanks

The authors 


\title{
A modelling approach for dedicated machinery to support the effects of mass customization
}

\author{
J Matthews $^{1}$, B Singh ${ }^{1}$, A Delandes ${ }^{2}$, G Mullineux ${ }^{1}$ and A. J Medland ${ }^{1}$ \\ ${ }^{1}$ Department of Mechanical Engineering, University of Bath, BA2 7AY \\ ${ }^{2}$ Universite de Technologie, 60206, Compiegne, Cedex
}

\begin{abstract}
In recent years the strategy of mass customization has become being increasingly adopted as manufacturers seek to exploit market trends for greater product variety and individualisation. The implications of changing to mass customization practice are considerable, specifically for the equipment producing the product. It is common practice within manufacturing companies to rely on trial-and-error approaches to develop their existing processing equipment for new or variant products. The approach presented in this paper employs the capability of a modelling environment to represent and simulate variations in machine design configuration and assess their ability to process variant products. The approach is demonstrated on an example from the packaging industry.
\end{abstract}

Keywords: Process modelling, manufacturing systems evolution, product variation, mass customization

\section{Introduction}

An array of new manufacturing techniques and operational practices has been gradually embraced, over the past decades, such as just-in-time manufacturing (Taiichi, 1988) and, later, variously, agile manufacturing (Dugary et al., 1997), lean manufacture (Womack et al., 1990). More recently the manufacturing sector has been driven by an increasing need to satisfy customer demands for greater product variety and for more responsive, small batch delivery. Mass customization has come to the fore (Tseng and Jiao, 2001). Products that are very similar in their general structure but differ in the details of each customer-specific variant can be grouped into general product families structures. Traditional dedicated (Koren, 1983) production machine design is directed towards the creation of a single configuration embodiment to meet a given specific performance requirements for a singular product (Hicks et al., 2001). The configuration of such machinery, whether from food processing, automotive sub-component assembly or electrical device sectors, is designed with an innate capability to handle slight variations in product (Matthews et al., 2007). This is initially achieved by simply providing tolerances to allow, for example, changes that occur in pack sizes to be accommodated, through user adjustments or complete sets of change parts. However when extreme conditions of setups or 
major changes in product size and configuration are considered, there is no guarantee that the existing machines are able to cope. The problem is even more difficult to deal with when completely new product families are proposed to be made on an existing product line.

Within the process and packaging industries, changes in product are often achieved through variation in packaging formats, numbers in a pack and the types of presentation employed, particularly in the supermarkets (Hanlon et al., 1998). All result in the supplier being forced to make more and more frequent changes to the line with little to no guidance on how this can be achieved (or even if it is at all possible). The lack of knowledge on the capabilities of the machines being used forces the supplier to undertake a series of practical product trials. These however can only be undertaken once the product form has been decided and produced. There is then little opportunity to make changes that could improve the potential output of the line and reduce waste. Hence, the design is only known to be able to accomplish the task for which it was originally intended. Its ability to perform related, variant tasks is uncertain. Work performed to investigate whether a configuration can produce a variant product, puts a high financial burden on companies already trading in a highly competitive sector. The research by Rigamonti and Tolio (2006) highlighted that, from a machine manufacturer's point of view, system configuration is the cornerstone in the offer of processing capability to their customers. But the authors noted that only $15 \%$ of such offers are followed by an order. So the configuration phase is an expensive task for the machine manufacturer. It is common in many industrial situations that companies are attempting to develop a range of machines to meet different customer's needs without a full understanding of the nature of their products and the global issues involved. There is thus a need for a supportive modelling approach that allows the effect of the variation in products produced by adoption of mass customization to be analyzed together with an understanding of the manufacturing machine capability. Only through analysis of its interaction can the capabilities of a machine be full understood and refined to make production possible.

\section{Background}

In order to investigate the effects of product variation, any approach must have certain attributes:

- the ability to parametrically model or simulate the configuration of the machine/ process;

- the ability to analyze simulations, (including motion); and

- the ability to investigate modes of failure.

This section reviews some contemporary approaches to provide these attributes. The most critical requirements for an engineer investigating the configuration of a machine and its capability to 2 
process a variant product is the ability to analyze configuration changes and their effects on the performance factors of machine and process. This section discusses previous approaches and applications that are open to the engineer. A variety of techniques for the positional and kinematic analysis of machine mechanisms has been documented (Shigley and Uicker, 1995); Dwivedy and Eberhard, 2006). These approaches have more recently been complemented by the use of computer simulations to model, analyze and simulate complex mechanisms. Such approaches can utilize standard packages for design though simulation, which are mainly employed to evaluate the performance capabilities of a particular configuration during the machine operation cycle (Woolfson and Pert, 1999). Contemporary CAD packages such as SolidEdge permit the designer to model and undertake motion analysis (both kinematic and dynamic), together with component interaction. CAE packages, such as BOSS Quattro (Radovcic and Remouchamps, 2002), offer the option to explore the design space by implementing parametric studies and provide sensitivity analysis to allow comparison of the effects of several parameters on chosen responses. Such packages can give the engineer the option to optimize selected effects of parameter change by combining methods from the design of experiments. Hicks et al., (2001) described a methodology using a constraint-based modelling environment for supporting and analyzing the design of packaging machinery at the embodiment stage. This method showed the ability of the modelling package to analyze the design of a mechanism.

The above methods analyze the design configuration and its motions. Another area of research, undertaken for the robotics industry forms the methods of understanding the limits of reach and motion for robots and manipulator type mechanisms in their configuration space (Pusey et al, 2003). This resultant volume (workspace) can be defined to represent the maximum limiting motion for the device. Curto et al. (2002) studied the design and workspace of a 6-6 cablesuspended parallel robot. They characterized the workspace volume as the set of points, which the centroid of the moving platform can reach with tensions in all suspension cables at a constant orientation. An approach has also been developed that uses an algorithm to plot clouds of points to represent the workspace boundaries of robot system (Kazerounian and Gupta, 1982). These defined the cloud boundaries and they are connected together to give the real workspace. Research by Botturi et al. (2003) devised a general method for workspace computation based on geometric sweep of spatial elements representing partial workspaces. The geometric algorithms developed by Gosselin et al. (1992) define the geometric boundary edges of a dexterous robot together with the total orientation, maximal and fixed orientation workspaces by considering the limits of the actuators. 
What is evident from the literature is there is a variety of underlying methods for machine assessment that could be applied to the configuration analysis for variant products. The simulation and modelling approaches identified above perform well in describing the physical geometrical extremes and configuration space of the mechanism and/or machine. They offer the user the ability to analyze motion and to explore the design space of a given system. If individual analysis tools and methods are employed for detailed investigation of a particular machine or mechanism, then the ability to generate optimum or best-performing design solution is severely frustrated (Hicks et al., 2001). With the tools and methods reviewed there are fundamental limitations, because of the following:

- They allow no consideration for other modes of failure or limits;

- The user is constrained by the functions offered by the respective system for modelling and simulation attributes;

- Even through, the user has modelled the design, the tool many not allow complete access to underlying constraints, which are fundamental to this approach.

With these factors in mind, it is evident that currently there is no single approach commercially available to answer questions about the ability of machine systems to deal with variant product configuration. This paper reports on the use of constraint-based techniques in this area. Such an approach offers more adaptability. It includes aspects of parametric modelling which is paramount to this approach. It allows motion and element interaction to be performed. Its inbuilt functions give the option of sensitivity analysis which together with the use of hard and soft constraint allows for optimization. The approach presented here, builds on previous constraintbased approaches to process flexibility (Matthews et al., 2006a) and limits modelling (Matthews et al., 2007a).

\section{Modelling with constraints}

In general there are several reasons for using modelling to investigate the capabilities of industrial machinery to process variant products. It provides the opportunity to experiment and analyze in a relatively low cost and low risk environment. In an industrial environment, the cost of getting it wrong is often very high and carries high risk. Changes to the real system are sometimes expensive, difficult or even impossible to achieve whilst the plant is in production.

The aim of a constraint-based modelling approach is to represent what is to be achieved, rather than how it is to be achieved. When an engineer encounters an existing design (produced by a 4 
previous designer) for the first time, knowledge of the design area is often ill-understood and the appropriate design rules are unclear. What are more apparent are the constraints which place limits upon the allowable forms of feasible design. These constraints may include performance and physical requirements of the design and also issues imposed by resources. In this manner, the design activities are not process led but goal orientated (Matthews et al., 2006b). When considering a manufacturing system it is vary rare that any single element or operation is independent of all the others. Consequently, all the goals must be dealt with concurrently and their relationships considered. The aim is to find a solution that satisfies all these imposed constraints as closely as possible. Search for acceptable design solutions is made using computational optimization techniques. The aim is to minimize the falseness of the improved constraints. This means that convergence on a fully successful solution is obtained if one exists or, if not, a best compromise is determined. This holistic approach allows the representation of design knowledge, and more importantly, enables this knowledge to be expanded or modified at any time during the process. In this way, changes are allowed in both the proposed solution and in the governing constraints of the particular design problem.

When constraints are used, there is a need to process the information they contain. Lin and Chen (2002) highlighted three types of constraint-based approaches employed for design and modelling. These are relevant across into the manufacturing domain. There are three levels of constraint-based approaches: constraint monitoring, constraint satisfaction and constraint optimization.

- Constraint monitoring uses constraints to check the proposed design solutions provided.

- Constraint satisfaction finds feasible solutions to constraints (without considering an optimal solution).

- Constraint optimization aims to find the best solutions form alternatives in order to achieve the objectives, subject to constraints; this requires some measure of performance of a solution.

These three handling approaches are illustrated in figure 1. This shows a contour plot of an objective function of two variables X1 and X2. Three linear constraints are imposed:

G0: $\mathrm{X} 2 \geq 0$

$\mathrm{G} 1: 3 \mathrm{X} 1+5 \mathrm{X} 2 \leq 30$ 
G2: $X 1-2 X 2+2 \geq 0$

These relate to the lines on the figure. The shaded triangle represents the feasible area. The unconstrained minimum of the function lies outside the triangle. The constrained minimum is at a vertex of the triangle. If the user employs constraint monitoring then each constraint is tested in turn. For constraint satisfaction, some point in the triangle is sought. Constraint optimization would find the constrained minimum (perhaps by traversing the boundary of the triangle).

\section{$<$ INSERT FIGURE 1>}

Figure 1 Constraint resolution strategies

With equipment design and development, the constraints can be applied at various levels. These are defined as hard and soft (Dechter, 2003) (cf. figure 2). Within this research, the hard constraints are concerned with function and assembly which ensure that the various parts of a system connect together correctly. At a higher level, the soft constraints can impose restrictions on performance such as kinematic properties. Additional constraints can relate to equipment cost and operation. Soft constraints may be weighted and may possibly violated, whereas the hard constraints must be true. The application of hard and soft constraints, is relevant to whichever constraint strategy the user employs i.e. constraint satisfaction, optimization or monitoring.

\section{$<$ INSERT FIGURE 2>}

Figure 2 Constraint pyramid

The language of the constraint modeller used in this research (Mullineux, 2001) has been created to handle design variables of several types including structured types to represent, for example, geometric objects. The language supports user defined functions that are essentially collections of commands which can be invoked when required. Input variables can be passed into a function and the function itself can return a single value or a sequence of values. An important inbuilt function is the "rule" command. Each rule command is associated with a constraint expression between design variables which is zero (as a real number) when true.

For constraint monitoring, logic rules (in the form of IF-THEN statements). For constraint satisfaction and optimization, the process involves varying a set of design parameters specified by the user. The sum is now treated as a function of these variables and a numerical technique is applied to search for values of the parameters which minimize the sum. If a minimum of zero can be found then the constraints are fully satisfied. If not, then the minimum represents some form of 6 
best compromise for a set of constraints in which there is conflict. Furthermore, it is possible to identify those constraints that are not satisfied and as a result less important constraints can be relaxed enabling an overall solution to be determined. The software environment supports simple wire-frame graphics, such as line segments and circular arcs. These can be defined in model spaces (Leigh, et al., 1989). A model space is a group of entities with which a transform is associated. This transform dictates how the entities map from their own local coordinates, into world space or into another model space. A model space can be embedded in another model space to create a hierarchy of spaces.

\section{Configuration modelling}

\section{$<$ INSERT FIGURE 3 NEAR HERE>}

Figure 3 Constraint-based model of ejection mechanism

As an example of the configuration of a mechanism in the constraint modelling environment, let us consider the ejection mechanism from a sweet wrapping machine. This cam driven mechanism's function is to guide the wrapped sweet from the transfer grippers onto a chute where the sweet exits the machine. The 3D representation of the whole mechanism can be seen in figure 3d. In part (a) of the figure, the two fixed pivot points 'G1' and 'G2' are specified, the closed curve representing the cam, and the line segments representing the four links are defined; the pushrod, the link, the ejection arm and cam follower. A point ' $\mathrm{P} 4$ ' is defined for the position of the cam follower on the cam follower arm. (represented by the dot on the cam follower). Initially points ' $\mathrm{G} 1$ ' and 'G2' are embedded in the machine model space. In the example, the model space of the pushrod and 'P4' are "embedded" in the space of the cam follower arm (M1). The spaces for the link and ejection arm are embedded in M2 together with the cam embedded in machine space. The hierarchy of these model spaces is shown in figure 4. A partial assembly of the mechanism is achieved by applying the transformations to the links in each space (this is shown in part (b) of the figure). If the space of either the crank or the coupler is rotated, the hierarchy of their spaces ensures their ends remain attached.

To complete the assembly, the ends of the pushrod and link have to be brought together; and the cam follower point 'P4' has to be put on the cam profile. This cannot be done by model space manipulation alone, as this would break the structure of the model space hierarchy. Instead a constraint rule is applied whose value represents the distance between the ends of the lines. The 
user language has a built-in function 'on' which returns the distance between its two geometric arguments.

To attach the cam follower to the cam profile rule ' $a$ ' is employed. With this rule invoked point 'P4' will follow the profile of the cam as the cam model space is transformed.

Rule 'A': $\quad \quad \quad r u l e(P 4$ on cam);

To attach the pushrod and link elements together, rule ' $b$ ' is imposed.

Rule 'B': $\quad$ rule ( pushrod:e2 on link:e1 );

The colon followed by e1 or e 2 denotes either the first or second end-point of the line. In order to resolve these constraint rules, the system is allowed to alter the angle of the model space of the cam. When the rule is applied then the correct assembly is obtained as in part (c) of figure 3. Constraint optimization is employed for this process. When the space of the cam is rotated and the assembly of the other elements is performed at each stage, a step-wise simulation of the motion is obtained. If solid objects representing the link are constructed, these can also be included in the model spaces as shown in part (d) of the figure.

\section{$<$ INSERT FIGURE 4 NEAR HERE $>$}

Figure 4 Hierarchy of model spaces for the ejection mechanism

With the mechanism defined, its functionality can be explored. There are instances when the mechanism functions correctly; conversely there are instances where the mechanism will fail. It is the exploration of the areas where the mechanism works or fails that allows the possibilities of creating designs that can cope with new product variation.

\section{Modelling for analysis}

Figure 5 summarizes, as a flow chart the methodology described above. The core of the flowchart show the steps of the method employed. The right shows how the stages of the method compare to conventional design and development activities. The left of the figure shows how the constraints are employed throughout the method. Firstly, with equipment redesign, the critical factor is the identification and formalization of the functional requirements for the redesign, in respect to the inherent capabilities of the existing design. With the requirements specified, the 
constraints imposed by the existing equipment and that of the product variation can be formalized for the design problem.

\section{$<$ INSERT FIGURE 5 NEAR HERE>}

Figure 5 Methodology

With the problem of investigating the effects of mass customization on a manufacturing system, one of the most critical aspects is the identification and formalization of a set of requirements for the new product with respect to the capabilities of the existing configuration. This often involves full production trials with the existing machine and specific testing and measurement of the new product. Once the requirements are established, constraints can be formalized for the design problem. This set of initial constraints is embodied in the existing configuration. Here the configuration is parametrically modelled using the constraint modelling package. This model is employed to analyze and evaluate performance bounds of the configuration. The resultant model is validated and verified. Validation of the model is the process of making sure the model represents reality, whereas the process of verification identifies that the model operates as the designer or customer intents. Validation is generally performed by comparing the simulation of the model against the high speed video footage of the real system. Full validation of the model is important as the model presents data upon which judgements relating to any potential redesign strategies are later based.

While investigating the effects of the variant product on systems, it has been found that most of the failures occur when the equipment reaches its limit points. For example, insufficient displacement is the achievable the required motions force the mechanism apart, or if accelerations are too high, vibrations and wear are induced. However product factors also affect the failure responses. For example, consider a mechanical gripper and transfer mechanism from a piece of equipment producing a frozen product. Marketing changes now mean the customer is offering to produce in a non frozen variant. This affects the mechanism in two ways: the package is softer, so less grip pressure can be applied, and the speed of transfer is limited due to potential deformation of product. The product constraints have to be imposed. Each product and its preparation or assembly process demands different characteristics in order that it can be produced and handled successfully. Due to the handling and transfer processes involved, the strength and resistance to damage or movement upon on the machine, for example a conveyor belt, may need to be assessed. Many of these characteristics need to be determined and studied if the capability of the plant to handle such product is to be understood. The information is collected via experimentation 9 
using relevant testing equipment. Some products, such as foodstuffs differ from most commercially manufactured products in the fact, that it is solely customer perception of product that matters. Customers' views of quality come from their senses, manufacturers employ taste and smell panels, to assess quality and define the constraints (Fisher et al, 2005). The physical measurements of the system are recorded in combination with high speed video footage of the equipment operating.

When starting machine investigations the manufacturer has to perform some testing of the new product to define its bounds. These are fed back into the system as geometric, kinematic and dynamic failure mode constraints. These are now employed in the redesign of the system. The design engineer can investigate alternative configurations of machine elements looking for a successful one that does not invoke any of the new failure mode constraints. Successful instances and modified variables modified are recorded along with performance values for each instance. The approach not only allows the user to investigate the effects of disturbing the existing configuration, but also permits the designer/ engineer to investigate the effects change parts or additions to the configuration that may be required if the constraints cannot be otherwise satisfied.

The next stage is to investigate the models function for the variant product. Within this research, the term "disturbance" implies the mean parametric variation of the variables defined in the model. These are used to find the successful instances of the model operating under the failure modes (constraint monitoring is used at this stage). These failure modes are the limits define by product and mechanisms function. Results of the modelling produce a list of successfully functioning points these are recorded individually to form the matrix. This matrix is the first form of representation of the functional points comes, which can have the performance factor associated with them. It can also be useful to have the data from the matrix in a more graphical representation such as cloud and surface plots or failure mode maps (Matthews et al., 2007b).

In most cases, it becomes obvious that there are, multiple configuration that will process individual products. At this stage the differing characteristics can be investigated simultaneously and compared against selected critical product characteristics. At this stage the designers and manufactures will have to evaluate which design solution they feel is best. Here a constraintbased optimization approach can be employed to find the optimal instance for the given product. 
It is also possible at this stage to reuse the modeller's sensitivity analysis function upon each configuration.

\section{Industrial case study}

The following is a typical example of the type of situation a manufacturer has to handle. The problem is the investigation into the capability of an existing packaging system to handle dual product changes: new lighter packaging medium and a geometrically different box with cost implications. The case study also highlights the capability of the constraint modelling approach to synthesis motion and analysis solutions for variant products. The equipment is used to wrap film around products such as food cartons or consumer goods. The basic action is in three parts: a portion of film is extracted from the roll and positioned above the product. The product is raised up through the film and the film wrapped around the sides and sealed. Finally the sides of the pack are folded and sealed. All function of this process, are controlled by a motor driven transmission mechanism, which consists of, five cam driven sub-mechanisms, which actuates the core functions of the overwrap process: the product in-feed, film draw, film guillotine, the tucking mechanism and, the machine carriage (product linear motion).five cam driven submechanisms. The manufacturer has to pack a new product. This raises three issues:

1. The product is geometrically taller than the existing product.

2. Environmental awareness means the packaging company is now required to pack with a thinner film. The mechanical handling properties of this product are 'weaker' than current films.

3. To make the new product cost effective the production rate is also required to be increased. Existing products run between 60 and 80 parts per minute. Ideally the manufacturer would like to produce at 120 parts per minute.

With the above in mind, can the new product be processed using the existing system?

\subsection{Modelling}

A parametric model of the transmission system was constructed within the constraint modelling environment, as described in section 3. Figure 5 shows wire frame models of the mechanism produced within the modeller. Part (a) shows a partial 2D CAD drawing of the system. (b) Side view, (c) plan view and (d) isometric. Effectively the system configuration is five cam driven sub-mechanisms, which actuate all the packing functions. Constraint optimisation is employed to 
maintain connectivity and relations as the system is activated, constraint monitoring is then used to check the optimisation and the correct functionality of the mechanism..

\section{$<$ INSERT FIGURE 6 NEAR HERE $>$}

Figure 6 Constraint model of system

Investigations of the model show four of the sub-mechanisms do not invoke any design laws i.e. cam transmission or pressure angles, and the kinematics values, when the operation speed is increased and additional displacements are applied for the new product. The limiting part of this system is the elevator mechanism. The mechanism is required to push the product through the packaging medium and into to the wrapping station. The elevator of the machine is required to return quickly to the start position, so as not to interact with other parts of the machine.

Figure 7 shows a wire frame model of the mechanism produced in the constraint modeller. Item 1 is the drive cam, 2 the cam follower, 3 cam follower pivot, 4 connection rod, 5 is the elevator block, (Constrained to move up and down) and 6 is the cam follower arm. Further constraints for the models function are the attachment of the cam follower on the pivot. The cam follower must remain in contact with the drive cam throughout motion and the connection rod maintains connectivity between cam follower and elevator block.

\section{$<$ INSERT FIGURE 7 NEAR HERE $>$}

Figure 7 Constraint model of elevator

\section{$<$ INSERT TABLE 1 NEAR HERE $>$}

To accept the new product, the product input conveyor could be lower, as the main pack heads on the top of the machine are fixed. This then requires the elevator to start at a lower position and make up the new displacement. As identified earlier, further constraints are identified for processing the new product. These are shown table 1. Because the up stoke of product through the packaging medium also de-reels the medium, tests showed that an acceleration of $2 \mathrm{~g}$ was sufficient without damaging the film. Engineers also had fixed a $2.5 \mathrm{~g}$ working limit on the acceleration of the moving parts of the system. Constraint monitoring was employed to check for constraint violations. Figure 8 a shows the displacement profile for elevator mechanism. Before considering any modification to this profile the functional constraints have to be considered (cf. table 1). The displacement distance is fixed as it is required to transfer the product from the base 12 
of the machine to the packing height. The start and stop points are also critical as they are timed with other sub mechanisms within the machine; the product is required to be in place by the time the cam has reached $150^{\circ}$. This leaves the position of the peak of the lift profile as the only factor that can be modified. The timing for the lift relates to the rotational movement of the cam. The lift profile can be described by a sinusoid. To adjust the peak position, the sinusoidal motion law was modified. This modification was calculated to give peak positions from $10^{\circ}$ to $150^{\circ}$ cam timing. Some of the modifications can be seen in Figure $8 \mathrm{~b}$.

\section{$<$ INSERT FIGURE 8 NEAR HERE $>$}

Figure 8 Displacements and cams

The points from the modified sinusoid are employed as the drive geometries for the end effecter of the elevator. As the elevator is moved, the model space of the cam is rotated. With each movement of the elevator, a point is transferred from the end of the cam follower into the cam model space. The points define the new cam and they can be joined using a B-Spline. Each cam profile is saved. Some of the multiple instances of the cams can be seen in plotted in figure $8(\mathrm{c})$. These files were read back into the modeller and then run as the drive cam, and constraint monitoring being employed to check for any constraint violations. The acceleration, jerk and velocities were then logged against each profile and compared.

\subsection{Results}

For visual representation, surface plots (figure 9) are constructed from the results of peak accelerations plotted against production speed (parts per minute). The acceleration is plotted for both up and down strokes of the elevator. Both acceleration limits are identified on the plot. For the up stroke only the lower $2 \mathrm{~g}$ product limit is identified, as this is dominant at this stage.

The results show that using a modified cam profile there is the possibility of producing product at 120 parts per minute and still keeping the up stoke acceleration below $2 \mathrm{~g}$ and the down stroke below $2.5 \mathrm{~g}$.

$<$ INSERT FIGURE 9 NEAR HERE $>$

Figure 9 Surface representation of results 
In an attempt to utilize the optimal cam profile $\left(82^{\circ}\right)$ for the elevator mechanism, and to add additional flexibility to the system for future products. Further investigations were performed using a new configuration: employing a 'piggy-backed' linear drive mechanism on the top of the elevator block. This has the effect of changing the overall displacement of the top point of the elevator block and also to change the acceleration profiles. A wire frame model of this addition mechanism can be seen represented in figure 10.

\section{$<$ INSERT FIGURE 10 NEAR HERE $>$}

Figure 10 Elevator add-on mechanism

Results from this investigation highlighted that for the single product change example, presented in this case study, show the effects on dynamics out weighed any gains over cam alteration and potential flexibility for the case study. But if further multiple variant products were required, this modification has some merit.

\section{Conclusion}

This paper has presented an approach using a constraint-based modelling environment to model and simulate variations of system design configuration and assess their ability to process variant products. The approach has been created via wide-ranging investigations with industry and incorporates the need to embody and manipulate design knowledge, model and analyze systems, and to provide support for the rapid development and investigation of existing design configurations.

The advantage of using a constraint-based approach comes when an engineer encounters an existing design for the first time, knowledge of the design area is often ill-understood and the appropriate design rules are unclear. What are more apparent are the constraints which place limits upon the allowable forms of feasible design. Also with equipment redesign, the critical factor is the identification and formalization of the functional requirements for the redesign, in respect to the inherent capabilities of the existing design. With the requirements specified, the constraints imposed by the existing equipment and that of the variant product can be formalized for the design problem. If the existing design cannot meet the necessary requirement of a variant product, the modeller can be used to modify the configuration to seek a successful solution.

The specific outcomes of the approach include the fact that it offers the opportunity to investigate the redesign of a machine to handle product variation. It also offers the ability to represent failure 14 
modes into a constraint-based model and hence simulate its action. The constraint-based approach offers the possibility of optimizing a design when resources are in conflict.

\section{Acknowledgement}

The work reported in this paper has been supported by Department for Environment Food and Rural Affairs and the Food Processing Faraday Knowledge Transfer Network, involving a large number of industrial collaborators. In particular, current research is being undertaken as part of the EPSRC Innovative Design and Manufacturing Research Centre at the University of Bath (reference GR/R67507/01). The authors gratefully express their thanks for the advice and support of all concerned.

\section{References}

Botturi, D., Fiorini, P and Martelli, S.A Geometric method for robot workspace computation Proceedings of ICARO3 - The 11th International Conference on Advanced Robotics 2003.

Curto, B., Moreno, V and Blanco, F. J. A general method for c-space evaluation and its application to articulated robots. IEEE Transactions on Robotics and Automation, 2002, 18(1), $24-31$.

Dechter, R (2003) Constraint Processing, Morgan Kaufmann Publishers. USA.

Dwivedy, S. K and Eberhard, P., Dynamic analysis of flexible manipulators, a literature review Mechanism and Machine Theory, 2006, 41(7), 749-777.

Duguay, C., Landry, S and Pasin, F. From mass production to flexible agile production, International Journal of Operations and Production Management, 1997 17(12), 1183-1197.

Fisher, C., Medland, A. J. and Mullineux, G. Design for food quality. Proceedings of the 15th International conference on Engineering Design. (ICED05). Melbourne 2005, Australia

Hanlon, J. F., Kelsey, R. J and Forcini, H. E. (1998) Handbook of package engineering. Technomic Publishing Company. USA. 
Hicks. B.J., Medland.A.J., Mullineux.G. A constraint-based approach to the modelling and analysis of packaging machine. Packaging technology and science 2001, 14, 209-225.

Gosselin, C.M., Lavoie, E., and Toutant, P. An Efficient Algorithm for the Graphical Representation of the Three-Dimensional Workspace of Parallel Manipulators, Proceedings of the ASME 22nd Biennial Mechanisms Conference, Scottsdale, AZ, USA, 1992, 45, 323-328.

Kazerounian, S. M. K. and Gupta, K. C. Synthesis of position generating crank-rocker or DragLink Mechanisms. Mechanism and Machine Theory, 1982 17, 243-247.

Koren Y., (1983) Computer Control of Manufacturing Systems, McGraw-Hill Book Company.

Leigh R.D., Medland A.J., Mullineux.G., and Potts I.R.B. Model spaces and their use in mechanism simulation. I. Mech. E part B .J.Eng. Manufact. 1989, 203, 167-74.

Matthews, J., Singh, B., Mullineux, G., Medland, A constraint-based approach to investigate the 'process flexibility' of food processing equipment. Journal of Computers and Industrial Engineering. 2006a, 51(4), 809-820.

Matthews, J., Singh, B., Mullineux, G., Medland, A.J and Hicks, B.J. Constraint modelling as a means for understanding the limitations of a design and its performance. Proceedings 6th International conference on Integrated design and Manufacturing in Mechanical Engineering. (IDMME) 2006b. Grenoble, France. Eds S. Tichkiewitch and M. Tollenaere. (12 pages on CD)

Matthews, J., Singh, B., Mullineux, G., Medland, A constraint-based limits modelling approach to investigate manufacturing machine design capability. strojniski.vestnik Journal of Mechanical Engineering, 2007a 53(7-8) 462- 477.

Matthews, J., Singh, B., Mullineux, G., Feldman, J and Medland, A.J. Islands of Failure in a sea of success: the use of failure mode maps to assess the capability of machines to handle product variation. Proceedings of International Conference on Engineering Design (ICED07), August 2007b Paris, France. ISBN: 1-904670-01-6 
Medland, A.J., Mullineux.G. and Rentoul. R.H. Integration of constraint and solid modellers. Proceedings of the International MATADOR Conferenece, March 1993.

Mullineux.G. Constraint resolution using optimization techniques. Computers \& Graphics, 2001, 25(3), 483-492

Rigamonti, $\mathrm{M}$ and Tolio, T. Process analysis and flexible transfer line configuration. Digital Enterprise Technologies 2006, DET2006, Portugal.

Pusey.J , Fattah.A, Agrawal.S and Messina.E . Design and workspace analysis of a 6-6 cablesuspended parallel robot. Procs IEEE/RSJ International Conference on Intelligent Robots and Systems, Las Vegas, NV, 2003.

Radovcic, Y and Remouchamps, A (2002) BOSS QUATTRO: an open system for parametric design. 23(2) Springer Berlin / Heidelberg.

Shigley, J. E and Uicker, J. J. (1995). Theory of Machines and mechanisms. McGraw-Hill International Editions.

Taiichi, O (1998) Just-In-Time for Today and Tomorrow, Productivity Press. ISBN 0-91529920-8

Tseng, M.M., Jiao, J. Mass customization, in: Handbook of Industrial Engineering, Technology and Operation Management, 2001, 3rd. ed., p.685; ISBN 0-471-33057-4

UDG SolidEdge, http://www.plm.automation.siemens.com/en_us/products/velocity/solidedge/ (accessed 20-03-08).

Woolfson, M. M and Pert, G. J. (1999). Introduction to computer simulation, Oxford University Press,

Womack, J. P., Jones, D. T. and Ross, D. (1990) The Machine that Change the World, Rawson Associates, New York. USA. 


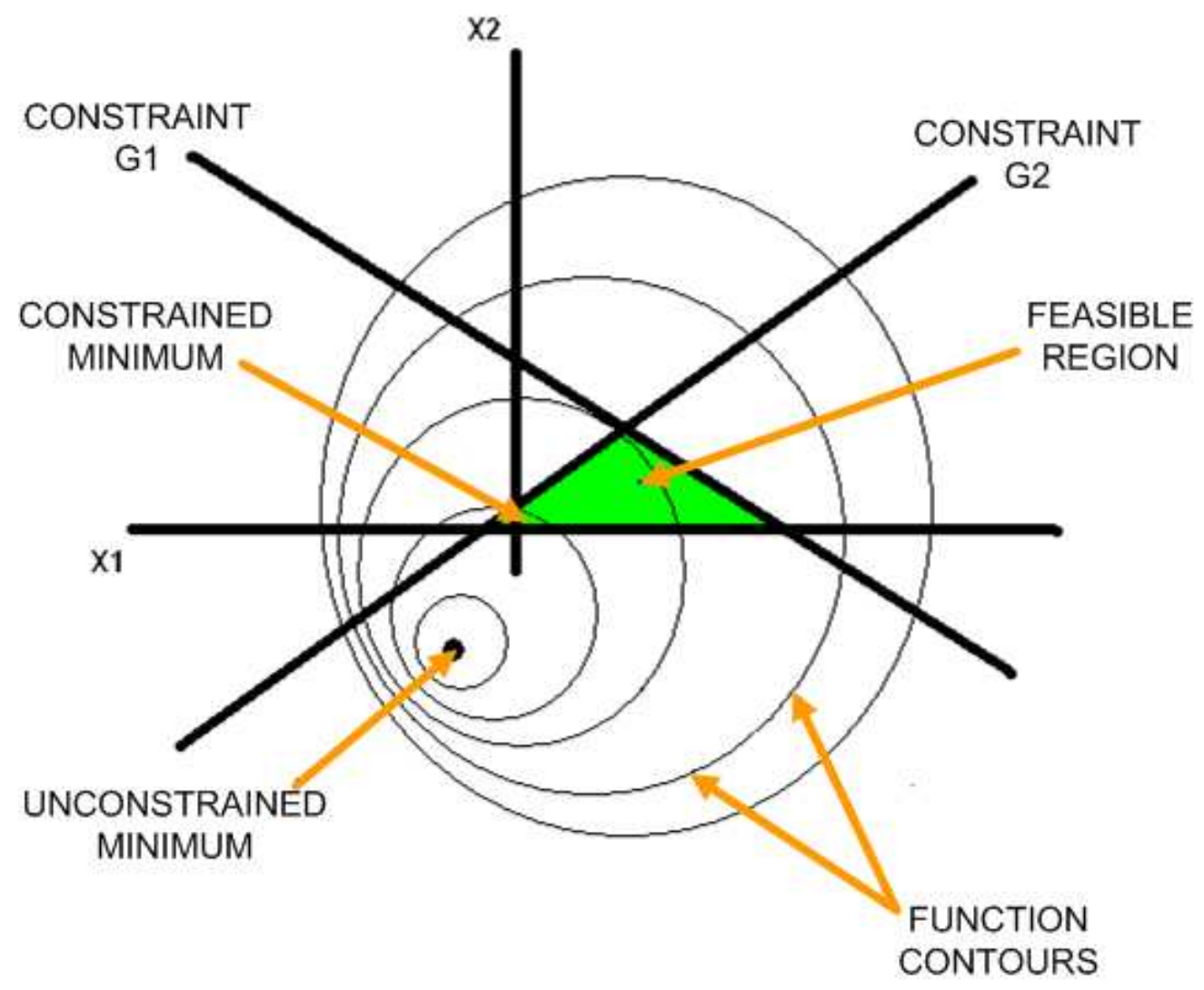

Constraint diagram

$143 \times 121 \mathrm{~mm}(96 \times 96$ DPI $)$ 


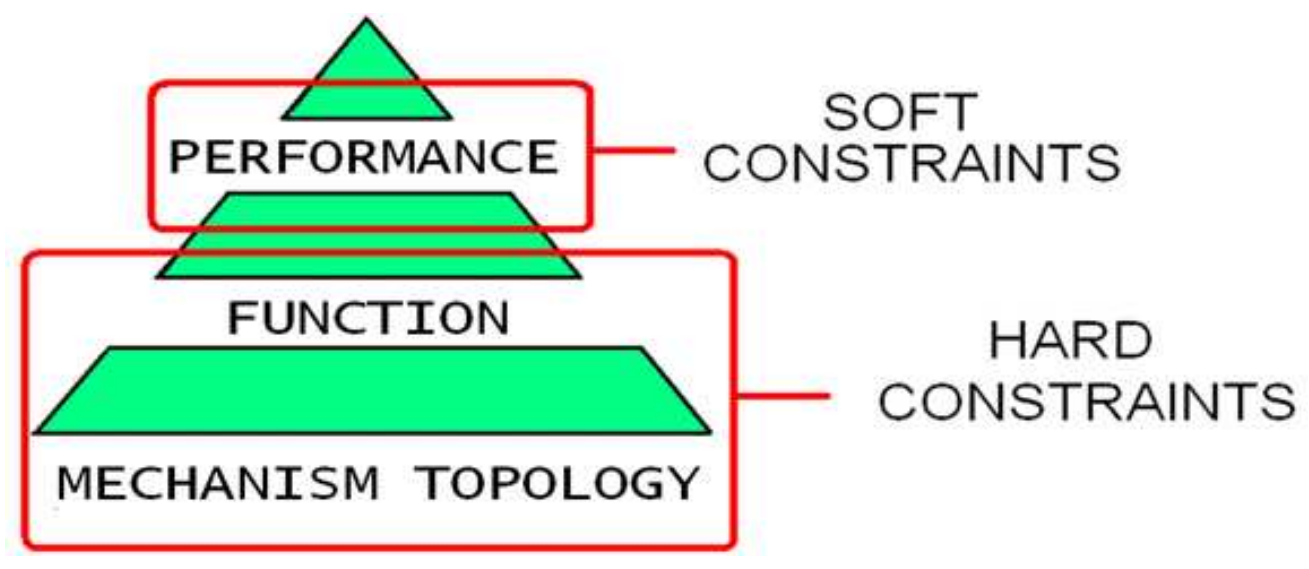

Constraint pyramid $152 \times 63 \mathrm{~mm}(96 \times 96 \mathrm{DPI})$ 


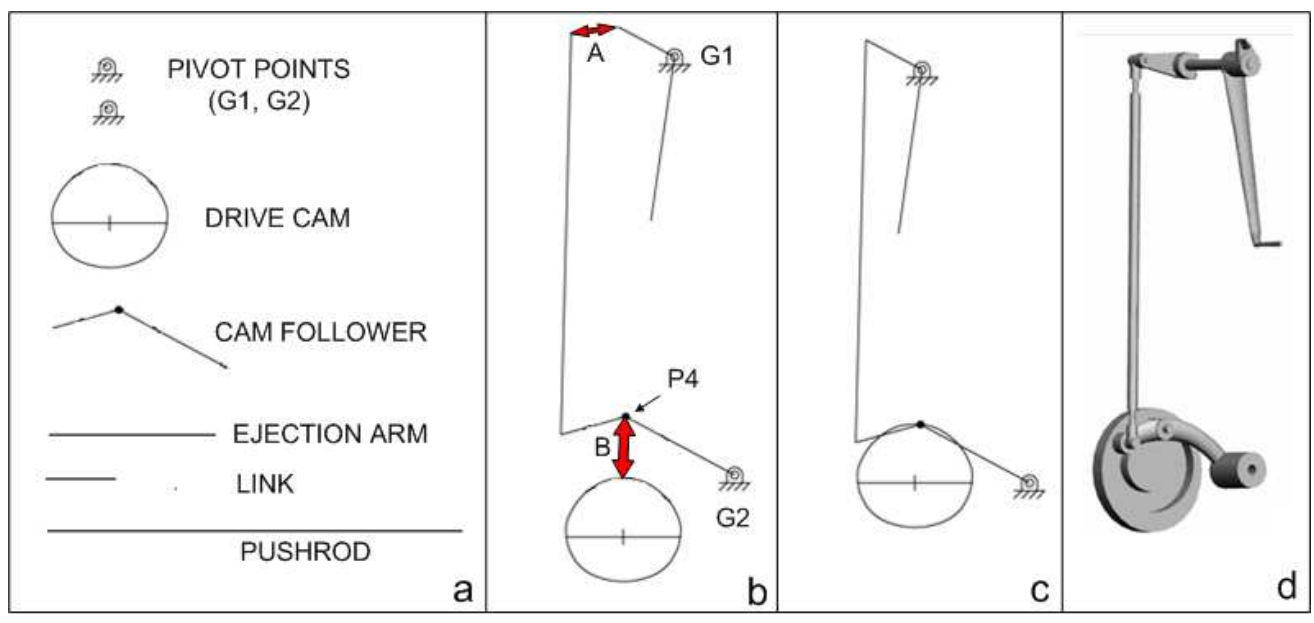

Construction of ejection mechanism $210 \times 98 \mathrm{~mm}(96 \times 96 \mathrm{DPI})$ 


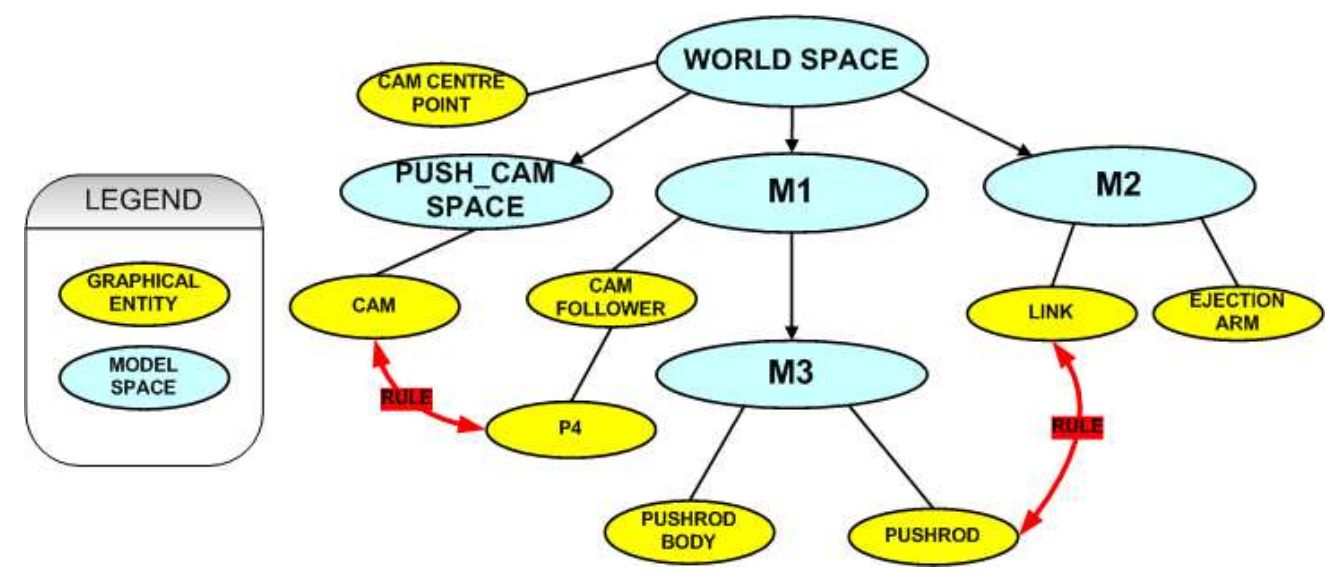

Model spaces

$192 \times 81 \mathrm{~mm}(96 \times 96 \mathrm{DPI})$ 


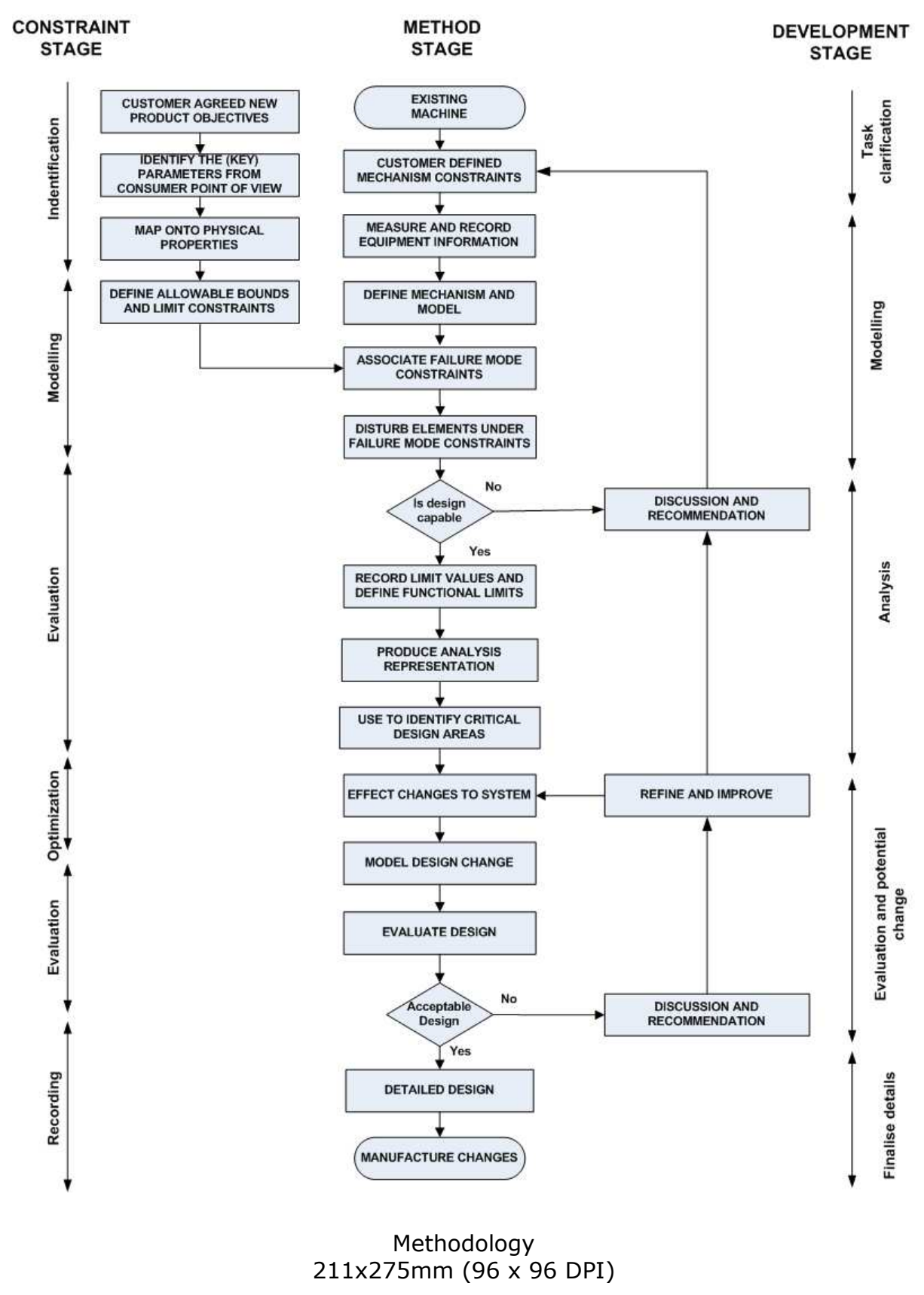

URL: http://mc.manuscriptcentral.com/tandf/tcim Email:ijcim@bath.ac.uk 


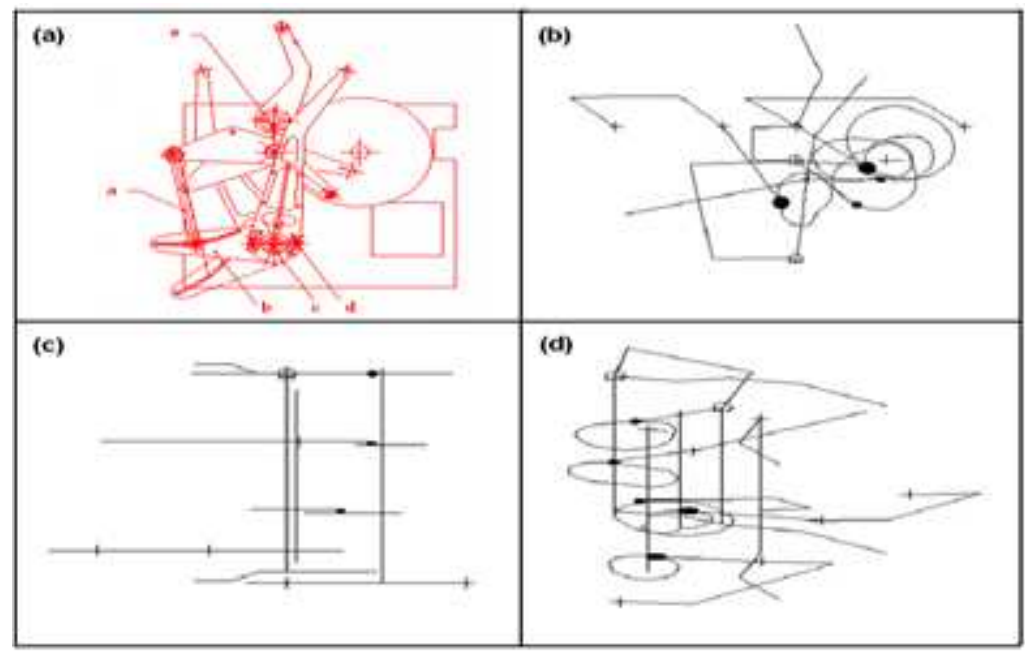

Figure 6 Constraint model of system $102 \times 65 \mathrm{~mm}$ (96 x 96 DPI) 


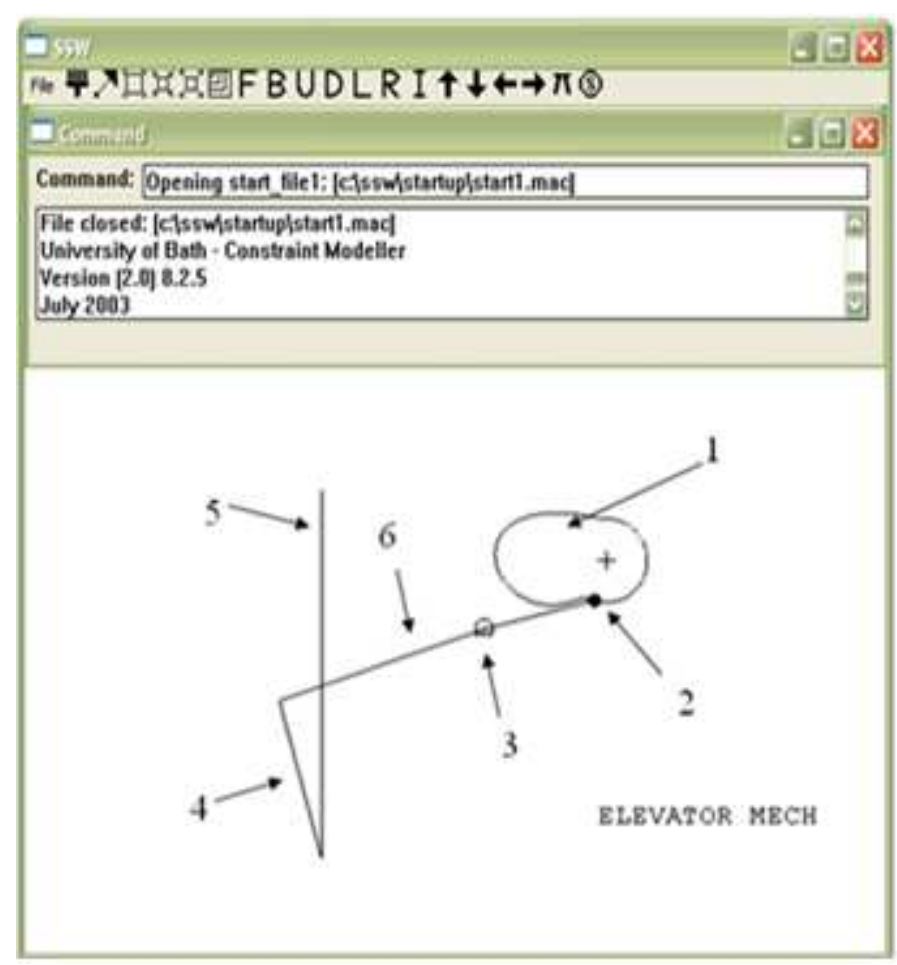

Figure 7 Constraint model of elevator $89 \times 96 \mathrm{~mm}(96 \times 96$ DPI) 


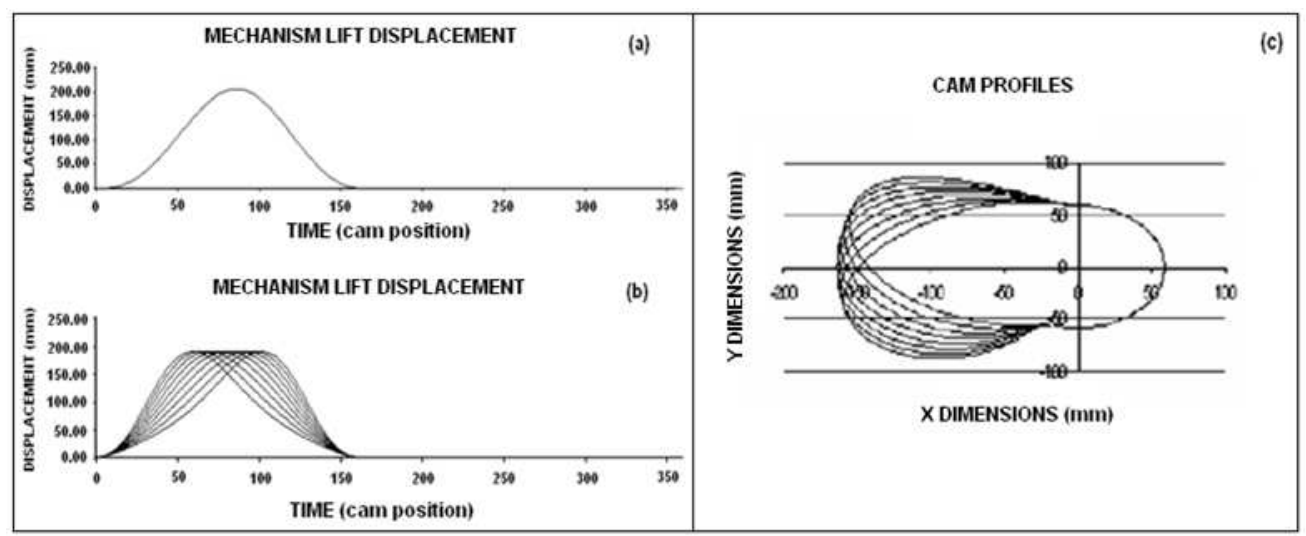

Figure 8 Displacements and cams 207x84mm (96 x 96 DPI) 


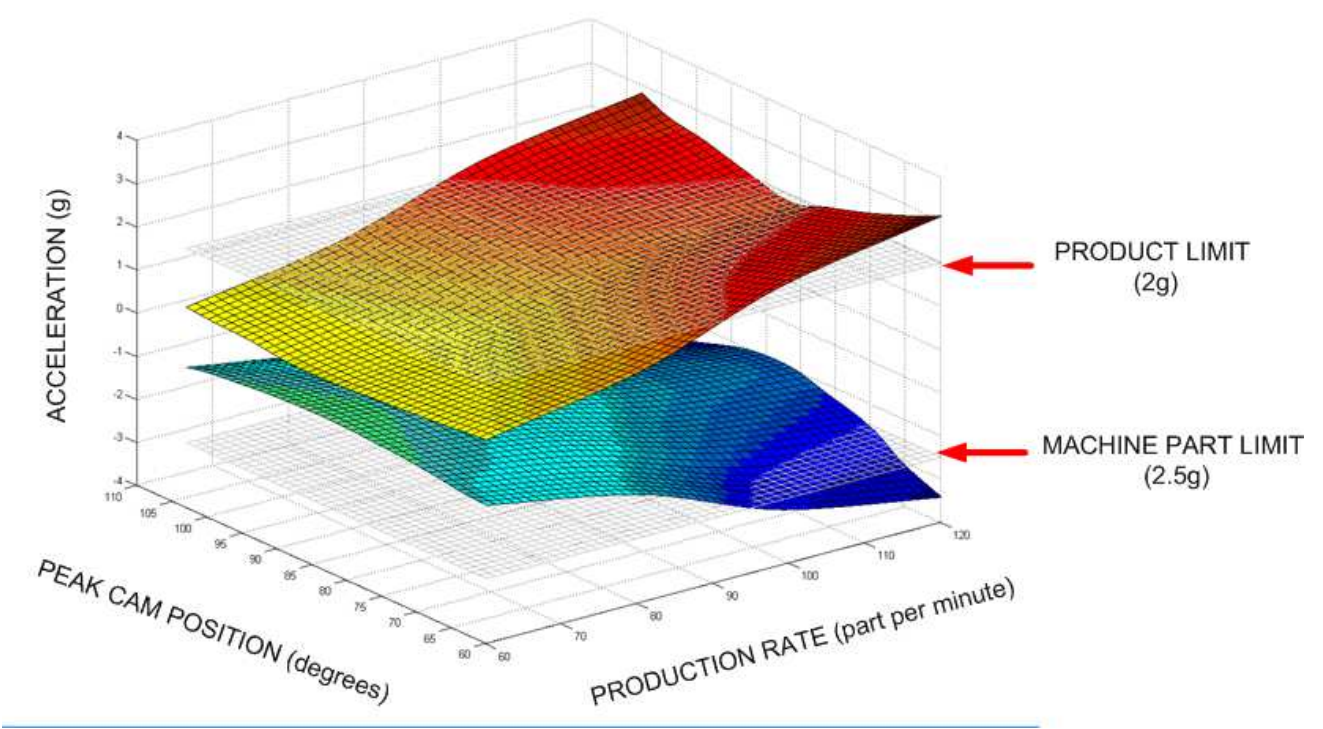

Figure 9 Surface representation of results $218 \times 128 \mathrm{~mm}$ (96 x 96 DPI) 


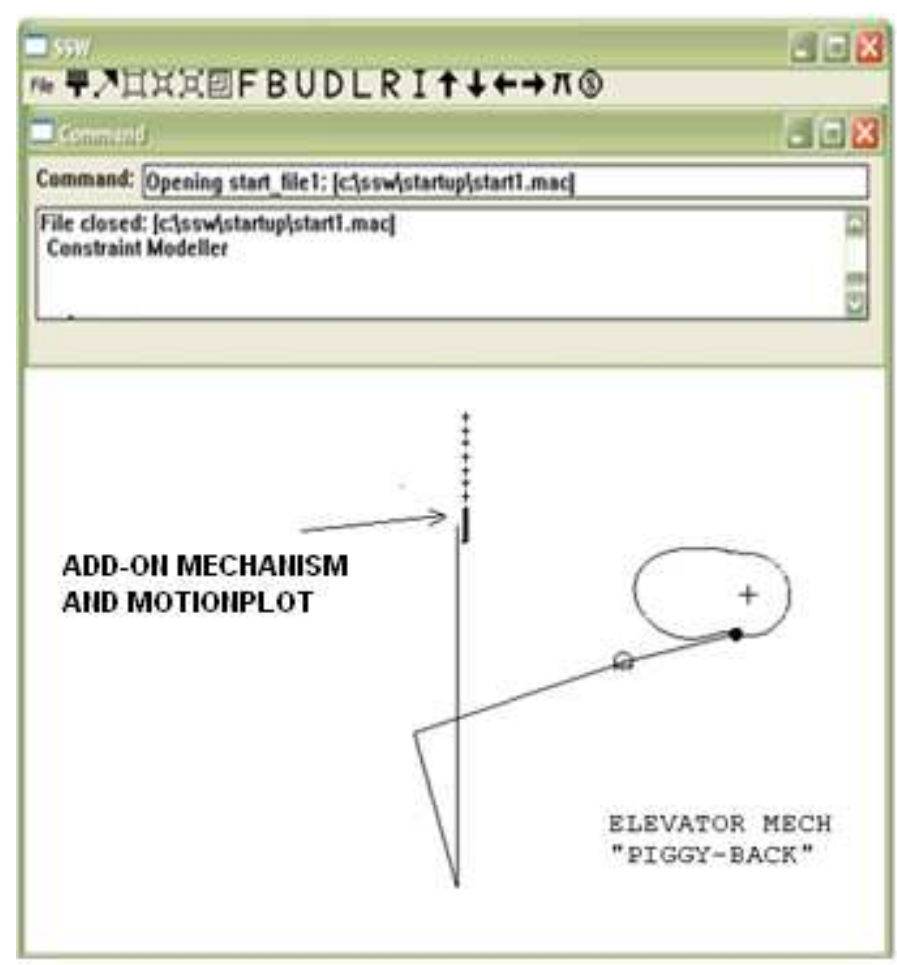

Figure 10 Elevator add-on mechanism $89 \times 96 \mathrm{~mm}(96 \times 96 \mathrm{DPI})$ 
Table 1 System failure mode constraints

\begin{tabular}{|c|c|c|}
\hline $\begin{array}{l}\text { Failure mode } \\
\text { constraint }\end{array}$ & Description & Association \\
\hline Geometric & $20 \mathrm{~mm}$ taller & Product \\
\hline Kinematics & $\begin{array}{c}2 \mathrm{~g} \text { limit of packing medium } \\
\text { acceleration }\end{array}$ & Product \\
\hline Kinematics & $\begin{array}{l}2.5 \mathrm{~g} \text { limit on mechanism } \\
\text { acceleration }\end{array}$ & Machine \\
\hline Timing & $10^{\circ}$ Cam Start position & Machine \\
\hline Timing & $150^{\circ}$ Cam finish position & Machine \\
\hline $\begin{array}{l}\text { Mechanism } \\
\text { deconstruction }\end{array}$ & Breakage in mechanism & Machine \\
\hline Geometric & $\begin{array}{l}\text { Element motion lie out side } \\
\text { machine footprint }\end{array}$ & Machine \\
\hline Collision & $\begin{array}{l}\text { Interaction of elements through } \\
\text { cycle of machine }\end{array}$ & Machine \\
\hline
\end{tabular}

